\title{
Registration of Ultrasound to CT Angiography of Kidneys: A Porcine Phantom Study
}

\author{
Jing Xiang ${ }^{a}$, Sean Gill ${ }^{c}$, Christopher Nguan ${ }^{d}$, Purang Abolmaesumi ${ }^{a}$ and Robert N. Rohling ${ }^{a, b}$ \\ ${ }^{a}$ Department of Electrical and Computer Engineering, University of British Columbia, \\ Vancouver, BC, Canada; \\ ${ }^{b}$ Department of Mechanical Engineering, University of British Columbia, Vancouver, BC, \\ Canada; \\ ${ }^{c}$ School of Computing, Queen's University, Kingston, ON, Canada; \\ ${ }^{d}$ Department of Urologic Sciences, University of British Columbia, Vancouver, BC, Canada
}

\begin{abstract}
3D ultrasound (US) to computed tomography (CT) registration is a topic of significant interest because it can potentially improve many minimally invasive procedures such as laparoscopic partial nephrectomy. Partial nephrectomy patients often receive preoperative CT angiography, which helps define the important structures of the kidney such as the vasculature. Intraoperatively, dynamic real-time imaging information can be captured using ultrasound and compared with the preoperative data. Providing accurate registration between the two modalities would enhance navigation and guidance for the surgeon. However, one of the major problems of developing and evaluating registration techniques is obtaining sufficiently accurate and realistic phantom data especially for soft tissue. We present a detailed procedure for constructing tissue phantoms using porcine kidneys, which incorporates contrast agent into the tissue such that the kidneys appear representative of in vivo human CT angiography. These phantoms are also imaged with US and resemble US images from human patients. We then perform registration on corresponding CT and US datasets using a simulation-based algorithm. The method simulates an US image from the CT, generating an intermediate modality that resembles ultrasound. This simulated US is then registered to the original US dataset. Embedded fiducial markers provide a gold standard for registration. Being able to test our registration method on realistic datasets facilitates the development of novel CT to US registration techniques such that we can generate an effective method for human studies.
\end{abstract}

Keywords: ultrasound guidance, image-guided therapy, partial nephrectomy, computed tomography, multimodality registration, intraoperative imaging, phantom studies, intensity-based registration

\section{INTRODUCTION}

Minimally invasive surgery often requires the acquisition of a 3D preoperative image with computed tomography (CT) which is used for planning. However, during the operation, many surgeons use ultrasound (US) to obtain real-time information. Using intraoperative ultrasound effectively is difficult since it contains significant artifacts and a limited field of view, unlike CT. Thus, obtaining a correct registration between the intraoperative ultrasound and the preoperative CT would improve navigation and guidance for the surgeon.

For further information, please contact:

Jing Xiang (E-mail: jxiang@ece.ubc.ca)

Robert N. Rohling (E-mail: rohling@ece.ubc.ca)

Robotics and Control Lab, Department of Electrical and Computer Engineering, University of British Columbia 2332 Main Mall, Vancouver, BC, Canada V6T 1Z4

Medical Imaging 2010: Visualization, Image-Guided Procedures, and Modeling, edited by Kenneth H. Wong, Michael I. Miga, Proc. of SPIE Vol. 7625, 762518 - @ 2010 SPIE · CCC code: 1605-7422/10/\$18 · doi: 10.1117/12.844594 


\subsection{Clinical Context}

Amongst Canadians, kidney cancer is the sixth most common cancer in men and the tenth most common in women [Canadian Urological Association 2007]. Similarly, it is among the ten most common cancers in men and women in the United States [American Cancer Society 2009]. The most common malignant tumour in kidneys is renal cell carcinoma (RCC). The standard of care for a patient who is suspected of having kidney cancer is to obtain a CT scan. The CT confirms the presence of the tumour and is used for surgical planning in the case that the tumour requires surgical resection. The patient often has CT angiography performed which involves getting a small dose of contrast agent injected intravenously at the time of the CT for the purposes of highlighting the vascular anatomy and the relation of the tumour to normal parenchyma. If the tumour is less than $7 \mathrm{~cm},{ }^{1}$ a section of the kidney containing the tumour can be removed. This laparoscopic partial nephrectomy procedure is preferred over radical nephrectomy because it controls the cancer while preserving renal function. Because laparoscopic cameras only allow surgeons to view the surface of tissues inside the peritoneum, visualization of the anatomy beneath including blood vessels and the target tumour would improve navigation during the surgery. CT provides a complete anatomical map of the patient but may be out of date, whereas US shows real-time updated images of the tissues but has a smaller field of view. The fusion of these modalities would be most useful. Recently, there has been some work published on registering video to $\mathrm{CT}^{2}$ and to $\mathrm{US}^{3}$ particularly for robot-assisted partial nephrectomy. Thus, the automatic registration of US to CT would make it possible to display both US and CT with video, which has the potential to improve the efficiency and success of these procedures.

\subsection{Related Work on CT to Ultrasound Registration}

Multimodality registration has been a topic of wide interest resulting in the development of many approaches to image registration of US to CT, and the similar problem of US to Magnetic Resonance Imaging (MRI). Several use a form of correlation ratio as the metric of comparison. Roche et al. perform image-based registration of 3DUS to MRI by using a bivariate correlation ratio metric that incorporates both intensity and gradient information. ${ }^{4}$ Leroy et al. also use correlation as the similarity metric except that they implement additional US preprocessing by removing speckle and shadow, and CT preprocessing by highlighting major boundaries. ${ }^{5}$ They optimize using the Powell-Brent Algorithm. While registration algorithms that employ correlation generally require moderate computational costs, the authors state that one of the problems is that their method is highly dependent on slice selection and the manually selected region of interest and initial starting point. Another approach to preprocessing the data is taken by Penney et al. whose goal is to register 3DUS to CT images of the femur and pelvis. The images are first converted from intensity images to probability images using a set a features. These probability representations are then compared using normalized cross correlation. ${ }^{6}$ It is important to note that the probability density functions are derived from training data. Thus, this approach requires a large amount of data and it is important to note that new data that does not resemble the training data may not register successfully.

Ultrasound to $\mathrm{CT}$ registration is difficult because the two modalities produce images with very different appearance, making it challenging to develop a metric for comparison. Recently, Wein et al. addressed this problem by simulating ultrasound images from the $\mathrm{CT}$ and then registering the original US to the simulated US. The authors use the basic physics of ultrasound to generate the simulated image. ${ }^{7,8}$ While the simulated US images are not completely realistic since they do not contain speckle and other artifacts, they allow for the registration of two image volumes with similar appearances. While the original method was tested on soft tissue such as kidney and liver, ${ }^{7}$ the method has also been applied to the registration of the lumbar spine. ${ }^{9,10}$ Further development has allowed for a computational speed increase by using a GPU-accelerated framework and the ability to register several US volumes. ${ }^{11}$

Of the above methods, the only studies that have a gold standard for validation are those performed on bone. Because Penney et al. performed registration on bone in cadavers, they were able to implant fiducial markers in the femur and pelvis. ${ }^{6}$ Similarly, Gill et al.'s phantoms of the spine also permit the implantation of fiducial markers. ${ }^{9}$ However, the studies of registration of soft tissue use human data and thus do not have a gold standard for validation. 

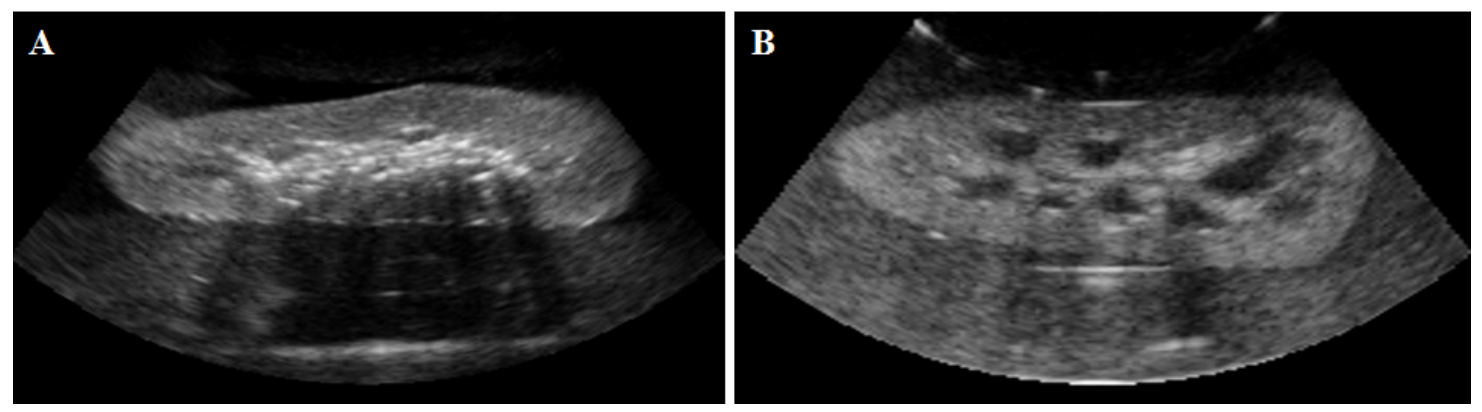

Figure 1. A) US of a grocery kidney with no preparation. B) US of a porcine kidney from a live pig.
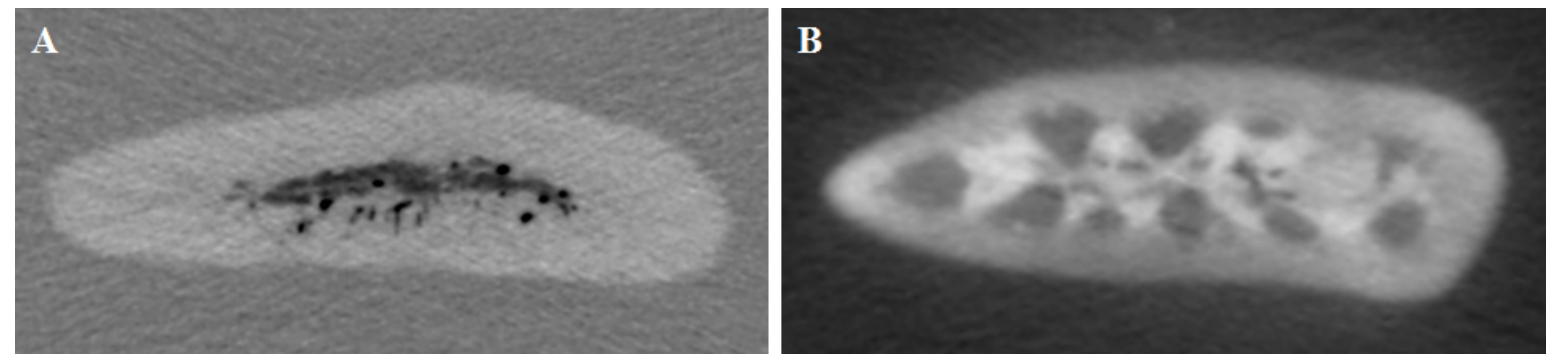

Figure 2. A) CT of a grocery kidney with no preparation. B) CT angiography of a porcine kidney from a live pig with contrast agent injected according to our protocol.

\subsection{Designing Accurate and Representative Phantoms for Registration}

One of the key challenges in developing US to CT registration is finding appropriate validation procedures. A popular method for validation of registration is to construct phantoms that model the structure of interest. However, creating a suitable phantom of soft tissue for a surgical application such as partial nephrectomy is difficult. These phantoms must appear realistic and be representative of the anatomy of interest in both US and CT imaging modalities. In this case, the phantoms must strive to accurately depict the kidney including the internal structures such as the renal pyramids and renal vasculature. For example, identifying landmarks in the vasculature may be useful for registration, ${ }^{12,13}$ which means that the structures must be visible in both modalities. At the present time, little work has been published on phantoms designed for US to CT registration. Recently, Cheung et al., also investigating registration for partial nephrectomy, used a phantom-less approach for validating the fusion of stereoscopic video and laparoscopic ultrasound. ${ }^{3}$ However, without the presence of kidney tissue in the image, it is not possible to discern features that are important to registration such as characteristic structures, average intensities of common regions, or realistic boundaries. We present here a detailed protocol for constructing a kidney phantom that produces high quality images in both US and CT angiography. The phantoms were designed not only to depict the surface boundaries of the kidney, but also to clearly define the vascular and pyramid anatomy of the kidney in both modalities. Since there has been recent success with a simulation-based registration approach, ${ }^{7,9}$ we will be testing the registration technique on our phantoms to evaluate its performance on aligning volumes of the kidney.

\section{METHODS}

When we began investigating the construction of soft tissue phantoms, we used kidneys obtained from butchers. However, getting high quality realistic images was difficult. Air inside the kidney created a problem for the US since it cast a large shadow in the US volumes (Fig. 1A). Because the renal hilum was removed, there was no method of introducing water or gelatin, and thus no means of injecting contrast. Therefore, it was difficult to distinguish between tissue and background in the CT (Fig. 2A). Thus, we eventually acquired kidneys that had their renal hilum intact from live pigs. Eight phantoms were prepared with agar and imaged with US and CT angiography. 

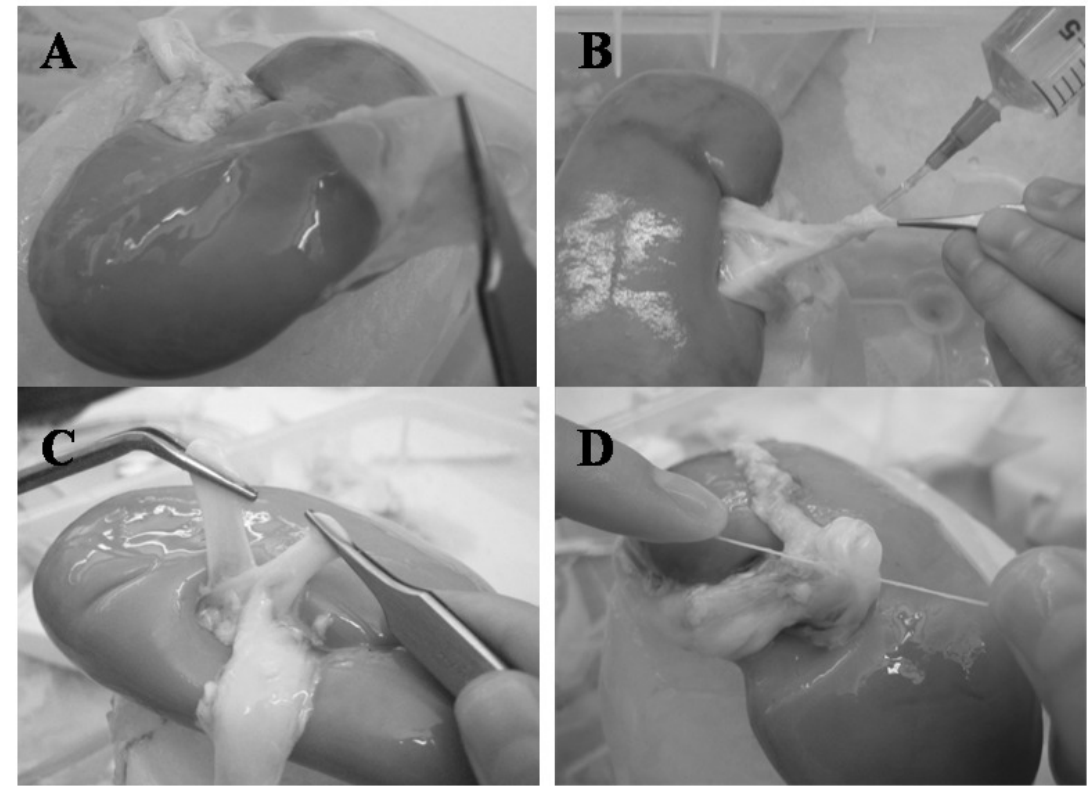

Figure 3. A) Porcine kidney during the removal of the renal capsule. B) Injection of diluted contrast agent into the renal artery. C) The dissected renal artery on the right and renal vein on the left. D) The renal artery and renal vein are tied off to prevent leakage of contrast agent.

\subsection{Procedure for Kidney Phantom Construction}

A plastic box was used to hold the kidneys which were enclosed in agar. The agar solution consisted of the following ingredients in percentage by mass: $1.17 \%$ high gel strength agar, $4 \%$ glycerol, $0.25 \%$ bleach, and $94.58 \%$ water. The amount of glycerol added was selected to regulate the speed of sound in agar such that it matches the typical speed of sound in soft tissue which is $1540 \mathrm{~m} / \mathrm{s}^{14}$ To prepare the agar, the reagents were mechanically stirred with a stir bar in a large beaker on a magnetic stir hot plate, and the temperature was brought to $90^{\circ} \mathrm{C}$. Stirring continued to ensure a uniform solution while the agar was allowed to cool at room temperature to $50^{\circ} \mathrm{C}$, prior to being placed in the refrigerator to solidify. A base layer was poured in the box first and allowed to harden.

Porcine kidneys were excised from live pigs from the Animal Laboratory at the Jack Bell Research Center (Vancouver General Hospital, Vancouver, BC, ethics certificate number A050316). The pigs were injected with heparin prior to excision to prevent clotting within the kidneys. The renal hilum and ureter were left attached and the renal artery and vein were dissected. Promptly after the kidneys were taken out, saline was flushed via a syringe through the renal artery. Flushing was repeated until the saline solution appeared clear when exiting the renal vein, indicating that most of the blood had been removed. The renal capsule was then removed so that it did not trap air bubbles that disrupt the US and CT images (Fig. 3A). Removal of the thin membrane had no noticeable effect on image features in either the CT or US. Two solutions containing the contrast agent Omnipaque iohexol (GE Healthcare, Mississauga, Ontario) injection (300 mg I/ml) were then prepared. First, $10 \mathrm{ml}$ of a 1 in 40 dilution in water was injected through the renal artery (Fig. 3B). Because the contrast was diluted with water, it diffuses into the parenchyma of the kidney and highlights the tissue in the CT image. Next, a $3.6 \%$ by mass gelatin solution was prepared by adding gelatin to water and heating until it dissolved. The solution was then cooled to $50^{\circ} \mathrm{C}$. For the second contrast solution, Omnipaque was diluted 1 to 5 by the gelatin solution. It is important to recognize that the second solution was more concentrated than the first and $10 \mathrm{ml}$ of it was injected into the kidney. Gelatin was used instead of water so that as the phantoms cool, the gelatin solidifies in the arteries, allowing us to highlight the arteries by not permitting the contrast to diffuse into the surrounding parenchyma. Finally, the artery and vein (Fig. 3C) were tied off (Fig. 3D) so that contrast agent does not diffuse into the surrounding agar. 


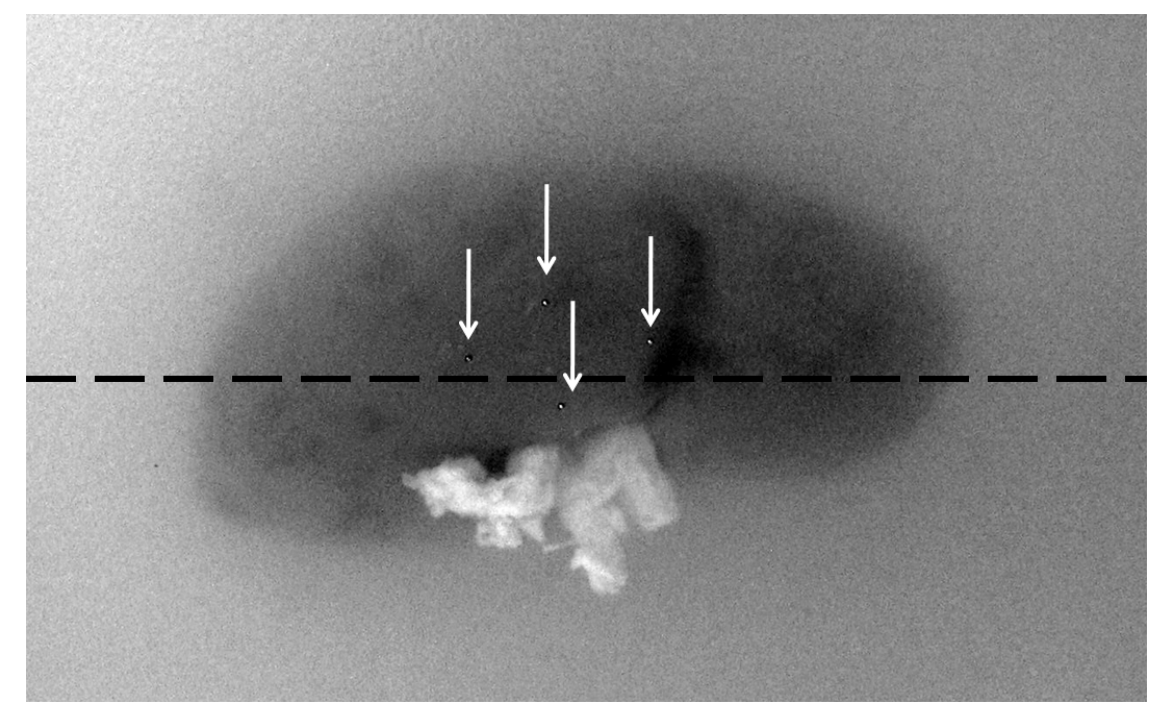

Figure 4. The locations of 4 steel ball fiducials are shown by the arrows. These fiducials are pressed into the agar. The dotted line represents the axis of the native scan. CT and US slices were taken parallel to that line.

Once the kidneys were properly prepared with contrast agent, two of them were placed on the agar base layer. The agar cooled to $50^{\circ} \mathrm{C}$, and was poured overtop until it covered the top of the tissue by about a centimeter. The box was then refrigerated until the agar had completely solidified. Next, four $1 \mathrm{~mm}$ stainless steel balls were pressed into the agar above each kidney (Fig. 4). These steel balls serve as fiducials which provided the gold standard for registration. More agar was poured over the steel balls to fill the rest of the box and the newly poured solution was cooled. There should be at least $25 \mathrm{~mm}$ of agar between the steel balls and the surface. This aids the 3D US acquisition as it ensures that the entire kidney is captured in the field of view of the sector-shaped volume.

\subsection{CT and Ultrasound Image Acquisition}

The CT scans were acquired using the Aquilion 64-slice CT scanner (Toshiba Medical Systems, Tustin, CA, USA) at $120 \mathrm{kVp}$. The pixel spacing was $0.468 \mathrm{~mm}$ and the slice thickness was $1 \mathrm{~mm}$. Pre-scan converted B-mode ultrasound volumes were obtained using the Ultrasonix Sonix RP machine (Ultrasonix Medical Corporation, Richmond, BC, Canada) with the 3-7 MHz convex curvilinear abdominal probe (4DC7-3). The 3D probe we used is a mechanical probe where the curvilinear transducer sweeps back and forth inside the case. The 3D probe securely fastened with a clamp above the kidney phantom. The $3 \mathrm{D}$ volume was then acquired at a frequency of $2.5 \mathrm{MHz}$ and a depth setting of $8 \mathrm{~cm}$, which was approximately the depth of the kidney. The US volumes were then scan-converted by using the geometry of the $3 \mathrm{D}$ probe. ${ }^{15}$

\subsection{CT and Ultrasound Image Registration}

Manual registration was performed using the locations of the four fiducial markers. To do this, the coordinates of the steel balls were found manually with a graphical user interface. Because the location of the bright intensity response in the US occurs at the top surface, the coordinates of the steel balls obtained represent the point on the top surface in both the CT and US. It has been shown that this approach will achieve sub-millimeter fiducial localization error. ${ }^{16}$ These points were used to calculate the six rigid registration parameters by using Horn's method. ${ }^{17}$ The rotation and translation in the $\mathrm{x}, \mathrm{y}$, and $\mathrm{z}$ direction were used to align the US and CT volumes, and was considered the gold standard. Image-based registration was then carried out on the corresponding volumes by using the approach proposed by Wein et al. ${ }^{7}$ with a Covariance Matrix Adaption-Evolution Strategy for optimization as it was proposed by Gill et al. ${ }^{9}$ This technique creates a simulated US from the CT and registers that image to the original US. The volumes were aligned at the gold standard before registration was performed. The CT volume was then perturbed by a transform selected randomly from a uniform distribution 

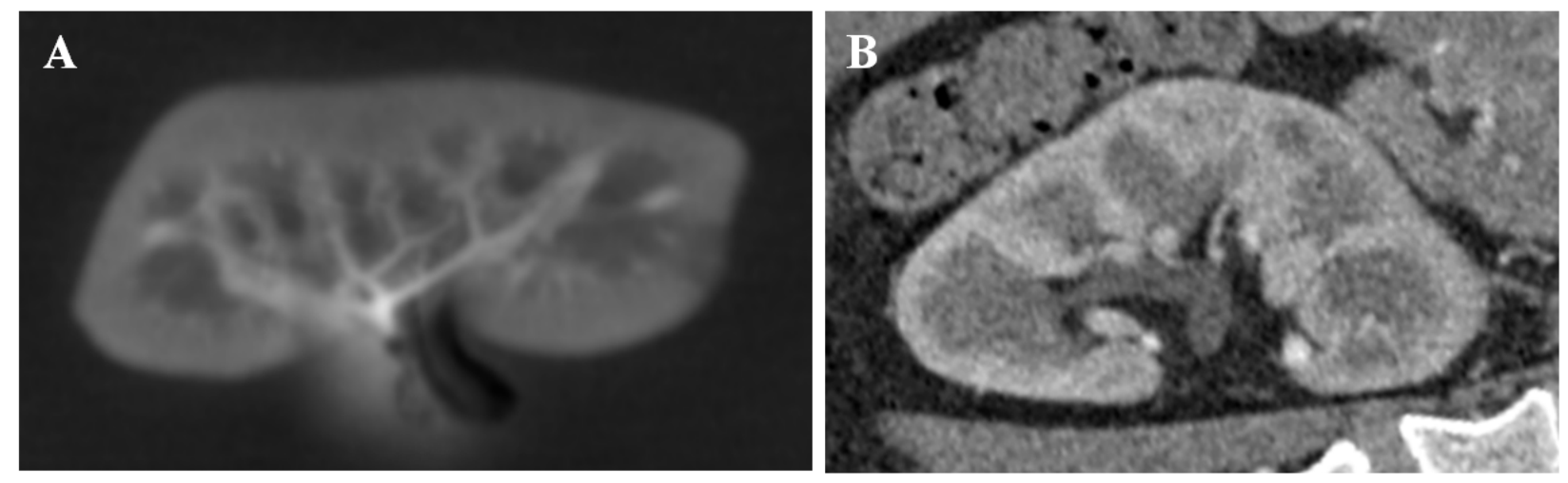

Figure 5. A) A coronal reconstruction of a CT angiogram acquired from a porcine kidney phantom showing internal structures of the organ. B) An example of a CT angiogram of a human kidney from a similar view is shown.
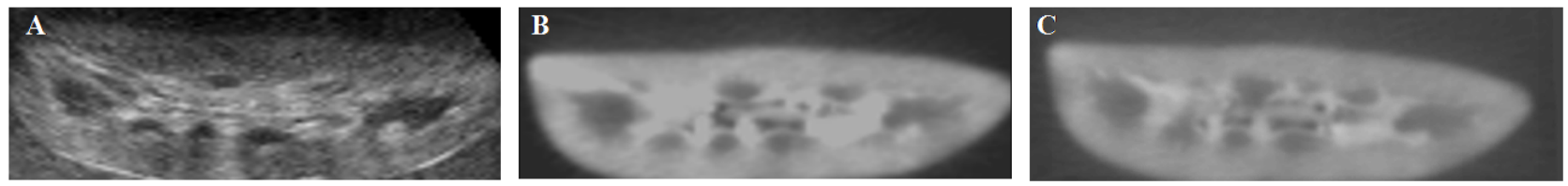

Figure 6. A) An transverse US slice of a porcine kidney phantom is shown. The simulated US is displayed in B) and C) shows the corresponding CT slice obtained by using the simulation-based registration algorithm.

of $10^{\circ}$ rotation about each axis and $10 \mathrm{~mm}$ translation along each axis. The target registration error ${ }^{18}$ (TRE) was determined from finding the misalignment of the corners of the volume.

\section{RESULTS}

The kidney phantoms that were created produced realistic US and CT images. The dark shadows in the US of porcine kidneys from the butcher (Fig. 1A) were corrected by harvesting kidneys from live pigs and preparing them with our protocol (Fig. 1B). In addition, after injecting contrast in kidneys from live pigs, the kidney tissue is distinctly different in intensity from the background agar, and the internal structure within the kidney is defined (Fig. 2B). From the coronal reconstruction (Fig. 5A), it is easy to identify the vascular system in the kidney, the renal pyramids that appear darker, and the renal cortex, the parenchyma tissue at the exterior of the kidney which defines the shape. This corresponds to the appearance of the human kidney in CT angiography as shown (Fig. 5B).

Thirty trials of the simulation-based registration were run on eight corresponding CT to US volumes. The last volume was excluded because there was an unexpected non-rigid distortion between the US and CT, and our rigid registration algorithm was unable to align the volumes. Therefore, seven out of the eight registrations generated a mean TRE of $5.71 \mathrm{~mm}$ with a standard deviation of $2.90 \mathrm{~mm}$. This is comparable to the mean TRE produced by registering a group of human liver and kidney datasets, performed by Wein et al. ${ }^{7}$ An example of a CT to US alignment by the simulation-based algorithm is shown below (Fig. 6).

\section{DISCUSSION}

This work presented a phantom design to create realistic images from porcine kidneys, for use in testing registration methods. We tested a simulation-based registration algorithm on the US and CT volumes acquired from these phantoms. The error in registration can be attributed to several sources. For our gold standard, we used 4 steel ball fiducials in each kidney phantom. The fiducials were placed far enough above the tissue so that they could be cropped out for image-based registration. Because the image depth of the US was set to the depth of the kidneys, there was lower fiducial resolution. Thus, there may have been error in locating the exact positions of the balls. This would result in a slight error in the gold standard. In the future, this should be remedied by 
taking identical volumes at two different depths, one at the depth of the fiducials and the other at the depth of the kidney.

In addition, we noticed a non-uniform deformation in the scan-converted 3D US volume. We suspect that the image deformations are caused by imperfect motor control on the particular probe that we used. Perfect motor control of the 3D probe would obtain each frame at an equal distance from the previous frame. The scan-conversion method used assumes this ideal case. In the future, we plan to verify the impact of this on our US volumes.

Enhancing the quality of US simulation from the CT may also improve registration results. For instance, our simulation did not consider the true shape of the radiating beam from the transducer when simulating the US image. In addition, we did not simulate speckle in the images. While these phenomena can be simulated using software packages such as Field II, ${ }^{19}$ it is extremely time consuming. However, it is possible to make some simple improvements to the algorithm such that the beam pattern is modeled. For example, the simulation can be modified to incorporate the effect of beam width as suggested by Sham et al. ${ }^{8}$

Because two layers of agar must be poured and must set after the kidneys are placed in the phantom, the phantom was prepared the night before the CT. Since we removed the renal capsule of the kidneys, contrast was free to leak out of the kidney tissue into the surrounding agar causing blurring around the boundaries of the organ. This reduced the image quality slightly which could potentially affect registration. It would be preferable to inject contrast at the time of the CT. It has been suggested that we stitch the artery to tubing that can be accessed from outside the agar layer. In this way, injection just prior to CT imaging would result in more crisp boundaries between tissue and background.

\section{CONCLUSION AND FUTURE WORK}

We presented a detailed recipe for constructing phantoms for the purpose of evaluating registration on kidneys. We prepared our kidneys with contrast agent so that they would reflect the appearance of in vivo human CT angiograms. They were then encased in agar medium so that we could obtain corresponding US images. Our gold standard was obtained by registering the volumes based on steel ball fiducials that appear in both the US and CT. We then used a slightly modified version of the simulation-based registration with CMA-ES optimization to register the US and CT volumes. Thirty trials were run which yielded a mean TRE of $5.71 \mathrm{~mm}$ with a standard deviation of $2.90 \mathrm{~mm}$. It can be argued that the simulated ultrasound appears very similar to the CT (Fig. 6). But, one visible difference is the shadowing that exists in ultrasound beneath the renal pyramids, is present in the simulated ultrasound yet absent in the CT. Thus, generating the simulated US does serve to modify the CT so that it resembles the US more closely. Because the internal structures are well defined in both modalities, it is possible that a feature-based approach would also produce good results. In addition, we recognize that ex vivo conditions will never be completely identical to the in vivo specimen. For instance, contrast agent is injected at the time of the CT in humans instead of hours prior in the phantom. Also, organ motion occurs in the human.

However, using these phantoms to test registration techniques provides first steps towards developing effective CT to ultrasound registration for human studies.

\section{ACKNOWLEDGMENTS}

We would like to thank Canada Diagnostic Centers and the Jack Bell Research Center for their technical support of this study. This research was supported by the National Sciences and Engineering Research Council of Canada.

\section{REFERENCES}

[1] Janetschek, G., "Laparoscopic partial nephrectomy: how far have we gone?," Current Opinion in Urology 17, 316-321 (2007).

[2] Su, L. M., Vagvolgyi, B. P., Agarwal, R., Reiley, C. E., Taylor, R. H., and Hager, G. D., "Augmented reality during robot-assisted laparoscopic partial nephrectomy: Toward real-time 3D-CT to stereoscopic video registration," Urology 73, 896-900 (2009). 
[3] Cheung, C., Wedlake, C., Moore, J., Pautler, S., Ahmad, A., and Peters, T., "Fusion of stereoscopic video and laparoscopic ultrasound for minimally invasive partial nephrectomy," in [Medical Imaging: Visualization, Image-Guided Procedures, and Modeling], Miga, M. I. and Wong, K. H., eds., Proceedings of SPIE 7261, 726109-1-726109-10 (2009).

[4] Roche, A., Pennec, X., Malandain, G., and Ayache, N., "Rigid registration of 3-D ultrasound with MR images: A new approach combining intensity and gradient information," IEEE Transactions on Medical Imaging 20(10), 1038-1049 (2001).

[5] Leroy, A., Mozer, P., Payan, Y., and Troccaz, J., "Intensity-based registration of freehand 3D ultrasound and CT-scan images of the kidney," International Journal of Computer Assisted Radiology and Surgery 2(1), 31-41 (2007).

[6] Penney, G. P., Barratt, D. C., Chan, C. S. K., Slomczykowski, M., Carter, T. J., Edwards, P. J., and J.Hawkes, D., "Cadaver validation of intensity-based ultrasound to CT registration," Medical Image Analysis 10, 385-395 (2006).

[7] Wein, W., Brunke, S., Khamene, A., Callstrom, M., and Navab, N., "Automatic CT-ultrasound registration for diagnostic imaging and image-guided intervention," Medical Image Analysis 12, 577-585 (2008).

[8] Shams, R., Hartley, R., and Navab, N., "Real-time simulation of medical ultrasound from CT images," Medical Image Computing and Computer Assisted Intervention 5242, 734-741 (2008).

[9] Gill, S., Mousavi, P., Fichtinger, G., Pichora, D., and Abolmaesumi, P., "Group-wise registration of ultrasound to CT images of human vertebrae," in [Medical Imaging: Visualization, Image-Guided Procedures, and Modeling], Miga, M. I. and Wong, K. H., eds., Proceedings of SPIE 7261, 72611O-1-72611O-9 (2009).

[10] Gill, S., Mousavi, P., Fichtinger, G., Chen, E., Boisvert, J., Pichora, D., and Abolmaesumi, P., "Biomechanically constrained groupwise US to CT registration of the lumbar spine," Medical Image Computing and Computer Assisted Intervention 5761, 803-810 (2009).

[11] Kutter, O., Wein, W., and Navab, N., "Multi-modal registration based ultrasound mosaicing," Medical Image Computing and Computer Assisted Intervention 5761, 763-770 (2009).

[12] Papenberg, N., Lange, T., Modersitzki, J., Schlag, P. M., and Fischer, B., "Image registration for CT and intra-operative ultrasound data of the liver," in [Medical Imaging: Visualization, Image-Guided Procedures, and Modeling], Miga, M. I. and Cleary, K. R., eds., Proceedings of SPIE 6918, 7691808-1-72611O-9 (2008).

[13] Lange, T., Papenberg, N., Heldmann, S., Modersitzki, J., Fischer, B., Lamecker, H., and Schlag, P. M., "3D ultrasound-CT registration of the liver using combined landmark-intensity information," International Journal of Computer Assisted Radiology and Surgery 4(1), 79-88 (2009).

[14] Madsen, E. L., Hobson, M. A., Shi, H., Varghese, T., and Frank, G., "Tissue-mimicking agar/gelatin materials for use in heterogeneous elastography phantoms," Physics in Medicine and Biology 50, 5597-5618 (2005).

[15] Pospisil, E., Zahiri-Azar, R., Rohling, R. N., and Salcudean, S. E., "Filtering and scan conversion of 3D displacement vectors from a 4D curvilinear transducer," Proceedings of IEEE International Ultrasonics Symposium, in press (2009).

[16] Hacihaliloglu, I., Hodgson, A. J., Abugharbieh, R., and Rohling, R. N., "Assessing surface localization accuracy in 3D local phase ultrasound images using CT reference images," 9th Annual meeting of the International Society for Computer Assisted Orthopaedic Surgery, 137-140 (2009).

[17] Horn, B. K., Hilden, H. M., and Negahdaripour, S., "Closed form solution of absolute orientation using unit quaternions," Journal of the Optical Society of America 4, 629-642 (1987).

[18] Fitzpatrick, J. M., West, J. B., and Maurer, C. R., "Derivation of expected registration error for point-based rigid-body registration," in [Medical Imaging: Image Processing], Hanson, K. M., ed., Proceedings of SPIE 3338, 16-27 (1998).

[19] Jensen, J. A., "FIELD: A program for simulating ultrasound systems," Medical \& Biological Engineering 8 Computing 34, 351-353 (1996). 